\title{
The 4D B-spline method of calculating left ventricular functional parameters of cardiac MRI to evaluate myocardial injury of the apical segment in patients with myocarditis: a case-controlled observational study
}

\author{
Xin-Xiang Zhao ${ }^{1 \#, ~ W e i-F e n g ~ Y u a n ~}{ }^{2 \# \wedge}$ \\ ${ }^{1}$ Department of Radiology, The Second Affiliated Hospital of Kunming Medical University, Kunming, China; ${ }^{2}$ Department of Radiology, The First \\ Affiliated Hospital of Chengdu Medical College, Chengdu, China
}

\#These authors contributed equally to this work.

Correspondence to: Wei-Feng Yuan, MD. Department of Radiology, The First Affiliated Hospital of Chengdu Medical College, 278 Baoguang Avenue, Xindu District, Chengdu 610500, China. Email: yuanweifeng3721@hotmail.com.

Background: Myocarditis does not have typical clinical manifestations and thus is difficult to accurately diagnose by virtue of infection history, and electrocardiogram (EKG) and peripheral blood abnormalities. Endomyocardial biopsy is the gold standard for diagnosis of myocarditis, but is invasive, high risk, and has an observational blind area. Cardiac magnetic resonance imaging (CMRI) is multiparameter and multidirectional with high spatial resolution and high contrast of soft tissue. However, the optimal method of calculating left ventricular (LV) function in patients with apical-segment-injured myocarditis is unresolved. We compared and analyzed the differences between two different methods (Simpson and 4D B-spline surface model (known as the 4D method)) of measuring LV function by CMRI in patients with myocarditis in the 17 th segment of the left ventricle.

Methods: The basic clinical data of two groups (myocarditis and non-myocarditis) were statistically analyzed, and differences in the LV function parameters by the two imaging methods were compared in the myocarditis group. Receiver-operating characteristic curves of single parameters and combined parameters based on the Simpson and 4D methods were drawn and the area under the curve, diagnostic threshold, maximum sensitivity interval, and maximum specificity interval were calculated.

Results: In the myocarditis and non-myocarditis groups the respective number of patients was 22 and 17 , the percentage of males was $54.55 \%$ and $47.06 \%$, and the average age was $32.20 \pm 11.59$ and $43.06 \pm 11.62$ years. The difference in $L V$ ejection fraction $(\mathrm{LVEF})(\mathrm{P}=0.033)$ and $\mathrm{LV}$ end systolic volume $(\mathrm{LVESV})(\mathrm{P}=0.030)$ in the myocarditis group was statistically significant. The respective AUCs based on the Simpson and 4D methods were LVEF 0.602 vs. 0.778, LVESV 0.556 vs. 0.751 , LVEF-and-LVESV 0.634 vs. 0.775. Based on the 4D method, the diagnostic thresholds of LVEF and LVESV were 34.965 (sensitivity 0.882 , specificity 0.591 ) and 69.090 (sensitivity 0.727 , specificity 0.706 ), the maximum sensitivity intervals of LVEF and LVESV were $(24.610,27.450)$ and $(35.355,37.200)$, and the maximum specificity intervals of LVEF and LVESV were $(60.530,65.625)$ and $(91.625,95.835)$, respectively.

Conclusions: Compared with the Simpson method, the 4D method might be more effective for CMRI diagnosis of apical-segment-injured myocarditis. When the Simpson method is used, LVEF combined with LVESV is recommended for comprehensive evaluation to improve diagnostic efficiency. When the 4D method is used, LVEF might be the preferred parameter for evaluation of LV function.

\footnotetext{
$\wedge$ ORCID: 0000-0001-8336-6822.
} 
Keywords: Heart disease; heart function tests; magnetic resonance imaging (MRI)

Submitted Feb 15, 2020. Accepted for publication Jul 21, 2020.

doi: 10.21037/qims-20-287

View this article at: http://dx.doi.org/10.21037/qims-20-287

\section{Introduction}

Myocarditis is localized or diffuse inflammation of myocardial tissue caused by a variety of inflammatory factors. Pathological changes include different manifestations of myocardial injury: degeneration and edema of myocardial cells, necrosis, infiltration of inflammatory cells in the myocardial interstitium, fibrosis, and so on. Severe heart failure and ventricular remodeling may occur in the later stage (1). The mortality rate of myocarditis is high, and research has shown that the proportion of myocarditis in cases of sudden death in adolescents is as high as $12 \%$ (2). However, myocarditis does not have typical clinical manifestations, so it is difficult to accurately diagnose by virtue of infection history, electrocardiography (EKG), and peripheral blood abnormalities only. Endomyocardial biopsy is considered the gold standard for the diagnosis of myocarditis, but is not widely used because it is invasive, high risk, and has an observation blind area (3). Cardiac magnetic resonance imaging (CMRI) is multiparameter and multidirectional with high spatial resolution and high contrast of soft tissue. It can directly observe pathological changes in myocardial tissue, providing an important basis for clinical evaluation of myocarditis, judgment of clinical stage, formulation of diagnosis and treatment plan, and evaluation of prognosis (4). T2 weighted imaging (T2WI), early gadolinium enhancement (EGE), and late gadolinium enhancement (LGE), are three basic sequences of CMRI for the diagnosis of myocarditis according to the Lake Louise Consensus Criteria (5), that are used to evaluate myocardial edema, capillary leakage, and fibrosis respectively. Some patients with myocarditis show left ventricular (LV) dysfunction, and approximately $9 \%$ of them each year will develop dilated cardiomyopathy (6). The Lake Louise Consensus Criteria also state that LV dysfunction on CMRI is indirect evidence supporting a diagnosis of myocarditis (5). However, there is no unified conclusion on how to measure this and which functional parameters are preferred. Currently, there are two methods of measurement: the Simpson method and the 4D B-spline surface model (known as the $4 \mathrm{D}$ method). The Simpson method is a set of $\mathrm{LV}$ functional parameter values of multiple groups (usually three, comprising the base, middle, and apex in 1 cardiac cycle) of a $3 \mathrm{D}$ short-axis dynamic cine sequence, which has gradually replaced the 2D linear static evaluation method in recent years. The premise is to simultaneously draw the annular lines of the endocardium and epicardium. However, for the short-axis position of the 17th segment (the apical segment), the endocardial annular line cannot be drawn, because the segment does not have a cavity, which is an observational blind area that can lead to inaccurate data. The 4D method is based on the traditional 3D Simpson method, with the addition of long-axis cine of the left ventricle. In addition, nine groups of short-axis positions of the base, middle, and apex are connected in series, which creates a more complete outline of the heart. Compared with the Simpson method, the 4D method may evaluate the systolic and diastolic function of the left ventricle more accurately and dynamically.

To date, there are only four articles published (7-10) on animal experimental modeling of the 4D method, and no clinical research reports in English. The aim of this study was to compare and analyze the differences between the two methods (Simpson and 4D) of measuring LV function by CMRI in patients with positive-sequence myocarditis in the 17th segment of the left ventricle. The structural and functional parameters of the left ventricle include $\mathrm{LV}$ ejection fraction (LVEF), fraction shortening (FS), LV enddiastolic dimension (LVEDD), LV end-diastolic volume (LVEDV), LV end systolic volume (LVESV), LV myocardial mass (LVMM), LV stroke volume (LVSV), cardiac output (CO), and cardiac index (CI). We discuss the optimal method of calculating LV function in patients with apicalsegment-injured myocarditis and the selection of single or combined parameters.

\section{Methods}

This was a case-controlled observational study conducted at the First Affiliated Hospital of Chengdu Medical College in China, from January 2018 to December 2019. The subjects, groups, inclusion criteria, and exclusion criteria are 
described below.

\section{Suspected myocarditis group}

The inclusion criteria for the suspected myocarditis group were the following: (I) presumptive clinical diagnosis of myocarditis in accordance with clinical signs and symptoms, including chest pain, exhaustion and palpitations, in addition to 24-h dynamic EKG abnormities; (II) negative computed tomography (CT) coronary angiography or negative $\mathrm{X}$-ray coronary angiography; (III) positive according to the Lake Louise Consensus Criteria; and (IV) apical segment (17th segment) identified as positive.

The exclusion criteria were the following: (I) claustrophobia or allergy to gadolinium (Gd-DTPA); (II) contraindication for MRI examination; and (III) serious arrhythmia or unable to trigger EKG gating.

\section{Myocarditis group}

This group was composed of patients with pathologically proven myocarditis from the suspected myocarditis group.

\section{Non-myocarditis group}

This group was composed of patients from the suspected myocarditis group who were pathologically shown not to have myocarditis.

All subjects gave informed consent, and all medical examinations were performed according to the guidelines of the Medical Ethics Committee of the First Affiliated Hospital of Chengdu Medical College. The authors also confirm that all ongoing and related trials are registered. The double blind method was used to minimize potential detection bias.

\section{Clinical data}

The number of cases, sex, mean age, clinical manifestation, coronary angiography, types of arrhythmia, and EKG were analyzed.

\section{Imaging of injured myocardial segment in the myocarditis group}

All magnetic resonance imaging (MRI) studies were performed with Siemens MAGNETOM Avanto 3.0T MRI equipped with high-performance gradients. Images were acquired in the four-chamber and two-chamber long-axis views with cine-MRI steady-state free precession sequences and in the short-axis plane with black-blood fast spin echo sequences, with double inversion recovery for suppression of the blood signal and an additional inversion recovery pulse for suppression of the fat signal to demonstrate the presence of feasible areas of high signal intensity given rise by edema. Next, a pile of continuous short-axis sections was acquired with cine-MRI sequences to assess global functional parameters of the left ventricle. After perfusion imaging, an additional dose of $0.1 \mathrm{mmol} / \mathrm{kg}$ of Gd-DPTA was administered at a rate of $2.0 \mathrm{~mL} / \mathrm{s}$. The EGE and LGE images were acquired after 2-3 and $10 \mathrm{~min}$, respectively, with breath-holding for each stack in the utilization of an IR-prepared and segmented gradient echo (GRE) sequence. The typical settings were the following: a pile of eight contiguous slices in the short-axis view with orientations identical to perfusion imaging, a slice thickness of $10 \mathrm{~mm}$ in the absence of an intersection gap, a TR/TE of $6.1 / 3.0 \mathrm{~ms}$, a field of view of $320 \mathrm{~mm}$, a matrix of $192 \times 160$, and a flip angle of $25^{\circ}$. Qualitative myocardial regional wall motion and cardiac function analyses were performed using a model of 17 segments in the short-axis orientation from the Target Map of the American Heart Association. The 17-segment model approach was used to define abnormal myocardial enhancement on LGE, and the presence of myocardial edema on T2-STIR and abnormalities on EGE were also described using the 17 -segment model. The total number and serial number of positive segments on T2WI, EGE, and LGE were observed. The location of enhancement within the myocardium was described as "subendocardial", "midwall", or "subepicardial", involving a variety of sections consisting of "basal", "middle", and "apex". All diagnoses were made strictly with reference to the Lake Louise Consensus Criteria, as described below (5).

(I) In the setting of clinically suspected myocarditis, CMR findings are consistent with myocardial inflammation if at least two of the following criteria are present [we called this a positive-sequence in our study]: (i) regional or global myocardial SI increase on T2WI; (ii) increased global myocardial early gadolinium enhancement ratio between myocardium and skeletal muscle on gadoliniumenhanced T1WI; (iii) at least 1 focal lesion with nonischemic regional distribution on inversion recovery-prepared gadolinium-enhanced T1WI.

(II) CMR study is consistent with myocyte injury and/or scar caused by myocardial inflammation if 
Criterion (iii) above is present.

(III) A repeat CMR study between 1 and 2-3weeks after the initial CMR study is recommended if (i) none of the criteria are present, but the onset of symptoms has been very recent and there is strong clinical evidence for myocardial inflammation; (ii) one of the criteria is present.

(IV) The presence of LV dysfunction or pericardial effusion provides additional, supportive evidence for myocarditis.

All CMRI images were analyzed by Syngo Argus software. The LV short-axis myocardium was divided into 17 segments, and three standard layers were identified, namely, the base, middle, and apex. The base and middle layers were divided into six muscle walls, and the apex was divided into four muscle walls (anterior, interior, inferior, and lateral) and the apex (i.e., 17th segment). Regional contractile function and biventricular global systolic functional parameters of the left ventricle (LVEF, FS, LVEDD, LVEDV, LVESV, LVMM, LVSV, CO, and CI) were calculated by the Simpson and 4D methods.

\section{Simpson method}

Using semi-automatic software, three groups (cine images of the cardiac cycle in one group of base, middle and apex) of short-axis endocardial and epicardial contours (i.e., the inner and outer rings) were manually depicted by mouse, and corrected and adjusted at the same time. After the LV contour was drawn, the height and weight of each subject were input, and the software automatically analyzed and generated the $\mathrm{LV}$ function curves and parameters.

\section{D method}

Based on the Simpson method, the two-chamber longaxis position was added and nine groups (cine images of a cardiac cycle in three groups of base, middle, and apex respectively) of the short-axis positions of the base, middle, and apex were connected in series.

Both the Simpson and 4D methods are slice-summation calculation methods for evaluation of regional and global $\mathrm{LV}$ function. All the results were reviewed by two human operators and the outlines of the endocardial and epicardial contours were corrected by semi-automatic correction software to achieve smooth distribution of the line between points along the endocardium and epicardium, and uniform line spacing without overlap or crossing.

\section{Statistical analysis}

Statistical analysis was done with SPSS 17.0 for Windows. All quantitative results are expressed in the text, figures, and tables. Analysis of variance (ANOVA) was used to analyze differences in $\mathrm{LV}$ function parameters between the myocarditis and non-myocarditis groups, and the results are shown as mean \pm standard deviation $(\mathrm{SD})$. We drew a receiver operating characteristics (ROC) curve for calculating the area under the curve (AUC) to evaluate the diagnostic efficiency and calculate the Youden index in order to determine the diagnostic threshold. A $\mathrm{P}$ value of $<0.05$ was considered statistically significant.

\section{Results}

\section{Clinical presentations}

A total of 102 patients were assessed for eligibility and of them, 45 were excluded for not meeting the inclusion criteria and 16 for not undergoing endomyocardial biopsy. Of the remaining patients, 22 with active lymphocytic myocarditis (T-lymphocyte was the main manifestation, but giant cells were not obvious) confirmed by histological and immunohistochemical examinations were selected, along with 19 patients without myocarditis confirmed by endomyocardial biopsy (Figure 1). The final number of patients in the myocarditis and non-myocarditis groups was 22 and 17 (2 patients were excluded from the nonmyocarditis group because of incomplete imaging data), the percentage of males was $54.55 \%$ and $47.06 \%$, and the average age was $32.20 \pm 11.59$ and $43.06 \pm 11.62$ years, respectively. In the myocarditis group, all patients had chest pain symptoms, and exhaustion and palpitation were present in $8 / 11$ and $1 / 2$ patients respectively; arrhythmias on $24-\mathrm{h}$ dynamic EKG findings ranged from tachycardia sinusale $(n=14)$, supraventricular premature beat $(n=12)$, ventricular premature contraction $(n=8)$, ventricular tachycardia $(n=8)$, and atrial fibrillation $(\mathrm{n}=1)$. All results are shown in Table 1.

\section{Cardiac MRI findings}

All 22 patients in the myocarditis group displayed a positivesequence (abnormal T2WI, EGE, or LGE), involving 93 segments in multiple locations of the myocardium (Table 2). The subepicardial myocardial region was the most frequent site of the positive-sequence (95\%), while midwall enhancement was less common (Figure 2). Involvement of the base, middle, and apex (serial numbers of the segments: 


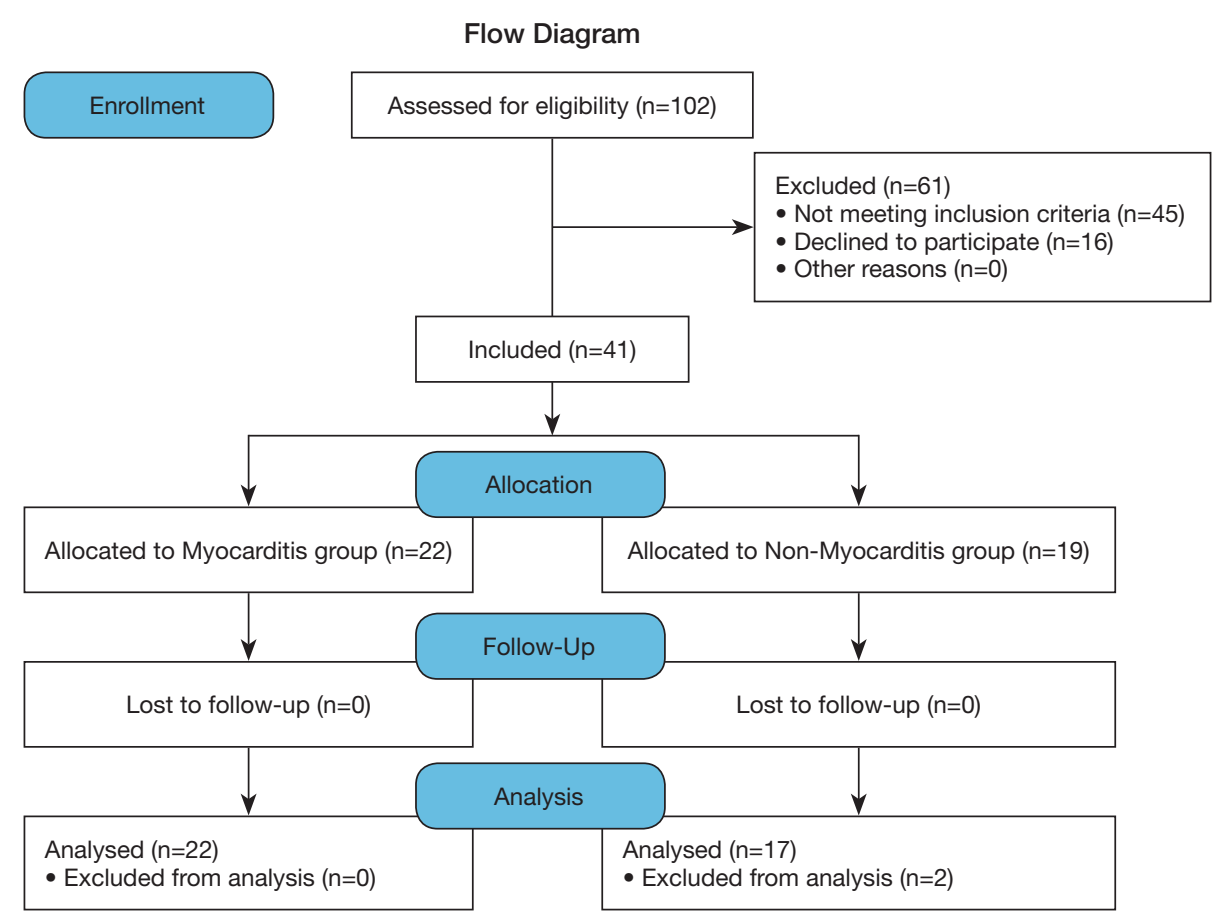

Figure 1 Of the 102 patients assessed for eligibility in this study, 45 patients were excluded for not meeting the inclusion criteria and 16 for not having endomyocardial biopsy data. The 22 patients with active lymphocytic myocarditis confirmed by the histological and immunohistochemical examination were selected as the Myocarditis group, and after excluding 2 patients due to incomplete clinical and imaging data, and 17 patients without myocarditis confirmed by the endomyocardial biopsy were selected as the Non-myocarditis group.

$2,3,4,5,6,11$, and 17) was found in a female patient presenting with a transmural (including subendocardial, midwall, and subepicardial) positive-sequence, but without an isolated subendocardial positive-sequence. The relevant curve and imaging for the 4D method were obtained by postprocessing software (Figure 3). Because some of the suspected myocarditis patients had been taking diuretics during hospitalization, and their weight and body surface area were undergoing constant and slight changes, we only included the absolute value and not the normalized value. The difference in LVEF $(41.76 \% \pm 11.16 \%$ vs. $34.52 \% \pm 10.66 \%)$ and LVESV $(64.24 \pm 18.48 v s .77 .15 \pm 19.58 \mathrm{~mL})$ was statistically significant $(\mathrm{P}<0.05)$ between the Simpson and 4D methods (Table 3). The difference in LVEF $(\mathrm{P}=0.033)$ and LVESV $(\mathrm{P}=0.0030)$ between the two methods was statistically significant in the myocarditis group. Based on the Simpson and 4D methods, the AUCs were the following: LVEF 0.602 vs. 0.778, LVESV 0.556 vs. 0.751, LVEF-and-LVESV 0.634 vs. 0.775 . Based on the $4 \mathrm{D}$ method, the diagnostic thresholds of LVEF and LVESV were the following: 34.965 (sensitivity 0.882 , specificity 0.591 ) and 69.090 (sensitivity 0.727 , specificity 0.706 ), the maximum sensitivity intervals of LVEF and LVESV were $(24.610,27.450)$ and (35.355, 37.200 ), and the maximum specificity intervals of LVEF and LVESV were $(60.530,65.625)$ and $(91.625,95.835)$, respectively (Figure 4).

\section{Discussion}

The heart is a complex system, integrating electrophysiology, mechanical dynamics, hemodynamics, energy metabolism, and cardiovascular regulation. By modeling and reconstructing the heart using medical data, we can analyze its different characteristics, such as geometry, electricity, and mechanical dynamics (11). The geometric/ anatomic model of the heart can be divided into a threedimensional or below three-dimensional static model or a four-dimensional motion model (12). The basic method of modeling is to segment certain medical data images to obtain a surface model, volume model, or deformable model of the heart. The CMRI cine imaging method has gradually become the gold standard for assessing cardiac morphological structure and function (13). CMRI quantifies LV systolic and diastolic function by measuring 
Table 1 Clinical characteristics in patients with pathologically proven myocarditis

\begin{tabular}{|c|c|c|c|c|c|c|}
\hline Case no. & Age (years) & Sex & Symptoms & Coronary angiography & Arrhythmias & EKG \\
\hline 1 & 41 & M & $\mathrm{C}, \mathrm{P}$ & N & TS, SPB & STE \\
\hline 2 & 18 & $\mathrm{~F}$ & $C, E, P$ & NA & TS, SPB & STD \\
\hline 3 & 37 & M & $\mathrm{C}, \mathrm{E}$ & NA & TS & NA \\
\hline 4 & 24 & $\mathrm{~F}$ & $\mathrm{C}$ & NA & NA & NA \\
\hline 5 & 45 & $M$ & $C, E, P$ & N & VPC, SPB & STE \\
\hline 6 & 34 & $M$ & $\mathrm{C}, \mathrm{E}$ & NA & $\mathrm{TS}, \mathrm{VT}$ & LBBB \\
\hline 7 & 38 & M & $\mathrm{C}, \mathrm{E}$ & NA & TS, SPB & STE, NT \\
\hline 8 & 26 & $\mathrm{~F}$ & $C, E, P$ & NA & TS & STD \\
\hline 9 & 18 & $\mathrm{~F}$ & $C, E, P$ & NA & NA & STE \\
\hline 10 & 45 & M & $\mathrm{C}, \mathrm{E}$ & NA & TS & N \\
\hline 11 & 27 & $\mathrm{~F}$ & $\mathrm{C}, \mathrm{P}$ & NA & TS, VPC, SPB & STD, NT \\
\hline 12 & 48 & M & $\mathrm{C}, \mathrm{P}$ & NA & TS & STE \\
\hline 13 & 11 & M & C & N & VT & RBBB \\
\hline 14 & 40 & $\mathrm{~F}$ & $\mathrm{C}, \mathrm{P}$ & NA & VT, VPC, SPB & STE, STD \\
\hline 15 & 34 & $\mathrm{~F}$ & $\mathrm{C}, \mathrm{E}$ & NA & AF & LAFB \\
\hline 16 & 37 & $\mathrm{~F}$ & $\mathrm{C}, \mathrm{E}$ & NA & NA & $\mathrm{N}$ \\
\hline 17 & 13 & $M$ & $\mathrm{C}, \mathrm{E}$ & NA & TS, VT, VPC, SPB & STD, NT \\
\hline 18 & 56 & M & $\mathrm{C}, \mathrm{E}, \mathrm{P}$ & N & VT, VPC, SPB & STD, NT \\
\hline 19 & 29 & $\mathrm{~F}$ & $C, E, P$ & NA & TS, VT, VPC, SPB & STD, NT \\
\hline 20 & 27 & $M$ & $\mathrm{C}, \mathrm{E}$ & NA & TS, VT, VPC, SPB & NA \\
\hline 21 & 39 & $\mathrm{~F}$ & $\mathrm{C}, \mathrm{E}$ & N & TS, SPB & STE, NT \\
\hline 22 & 28 & M & $C, E, P$ & NA & TS, VT, VPC, SPB & STD, NT \\
\hline
\end{tabular}

C, chest pain; E, exhaustion; P, palpitation; AF, atrial fibrillation; TS, tachycardia sinusale; VT, ventricular tachycardia; VPC, ventricular premature contraction; SPB, supraventricular premature beat; N, normal; NA, not available.

changes in LV volume during the cardiac cycle. At present, the Simpson method is commonly used for calculation of functional parameters, because it has better operability and repeatability in the determination of $L V$ volume and mass (14); that is, it is better at facilitating the drawing of endocardial and epicardial contour lines of the LV shortaxis layers in different cardiac cycles manually or semiautomatically. The absolute volume of the left ventricle is calculated by superposing the area of each layer. The parameters of $\mathrm{LV}$ function are measured by the change in volume in different phases. However, the Simpson method only obtains the volume of the heart through the shortaxis position of the left ventricle, so the root of the aorta often interferes with evaluation of images of the base; also, because the apical segment has almost no cavity structure, its endocardial margin cannot be drawn. Thus, the difficulty with accurately drawing the whole contour of the apical segment and the endocardial margin of the base can lead to bias in the measurement results. The 4D B-spline surface model (4D method) can define continuous surfaces at any spatial resolution, including the parametric description of the target or its motion state. It is mainly used for overall motion extraction of a small number of parameter representations (15). For example, Chandrashekara and others used the 4D method to track heart motion in tagged MR images (16). In clinic, the normal range of CMRI parameters used for evaluation of $\mathrm{LV}$ function has not been completely unified, and clinical research often uses LVEF 
Table 2 Characteristics of segment with myocardial injury in patients with pathologically proven myocarditis

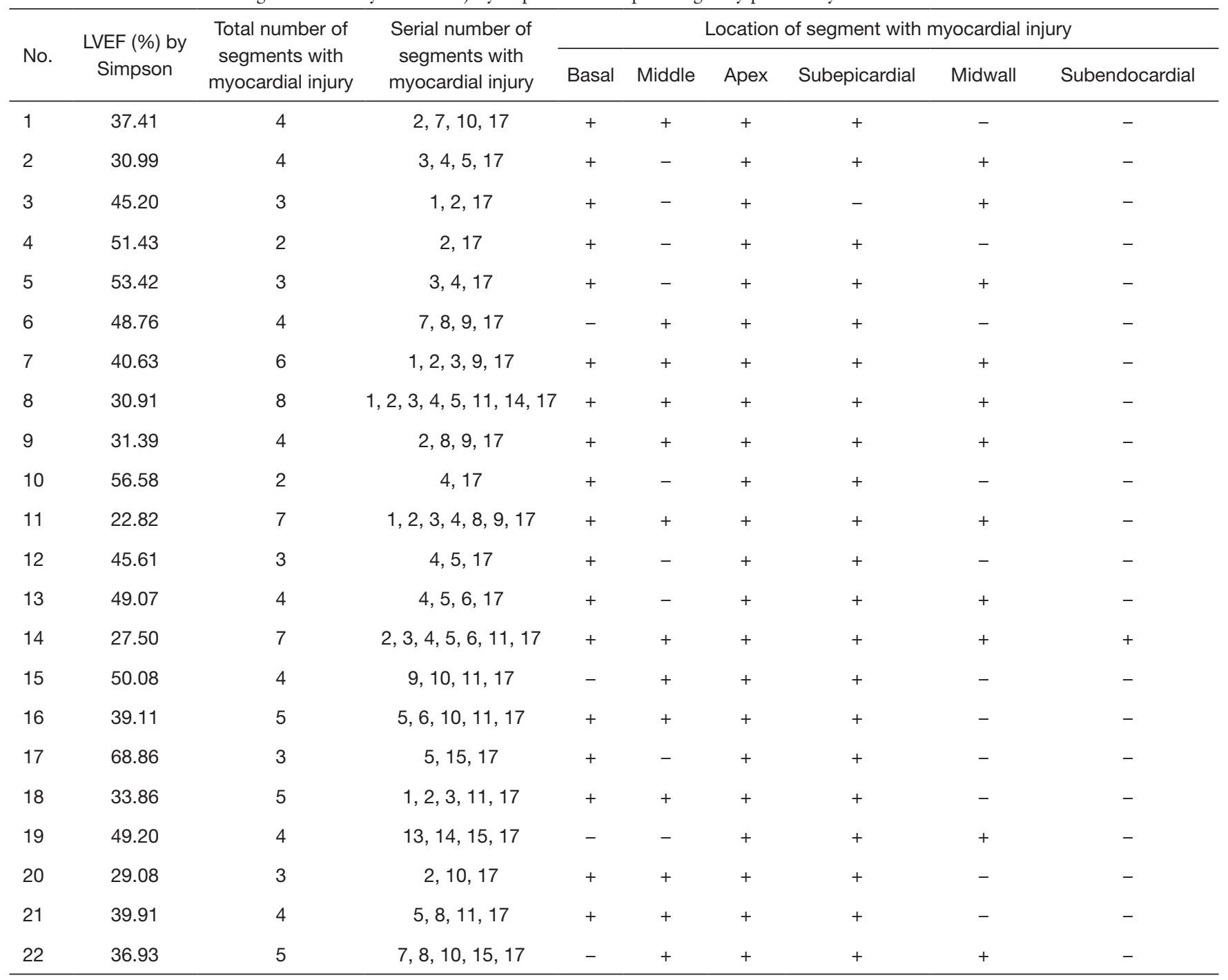

+ , positive; -, negative.

$>50 \%$ as the normal value range; therefore, the application of CMRI in the diagnosis of myocarditis needs further study. A few animal experimental models of the 4D method/ experimental research have been reported (7-10), but there are no clinical research reports in English. We previously researched the $L V$ function of healthy volunteers using the 4D method and 3D Simpson method, and our results suggested that the 4D method has the advantages of higher accuracy, greater repeatability, superior efficiency, and better intuition (17).

In this study, a CMRI post-processing workstation was used to quantize the original imaging data based on the $4 \mathrm{D}$ method, the height and weight of each subject were recorded to achieve standardization, and thus nine parameters of $\mathrm{LV}$ function were obtained. All diagnoses were made strictly with reference to the Lake Louise Consensus Criteria (5), and the CMRI findings were consistent with myocardial inflammation. Data from 22 patients with apical-segment-injured myocarditis were analyzed by oneway ANOVA of the structural and functional indexes of the left ventricle obtained using the two measurement methods. We found that LVEF and LVESV showed statistical difference, which suggested that they are more important than the other parameters in evaluating the structural and functional status of the left ventricle in patients with apicalsegment-injured myocarditis. Therefore, the 4D LVEF 

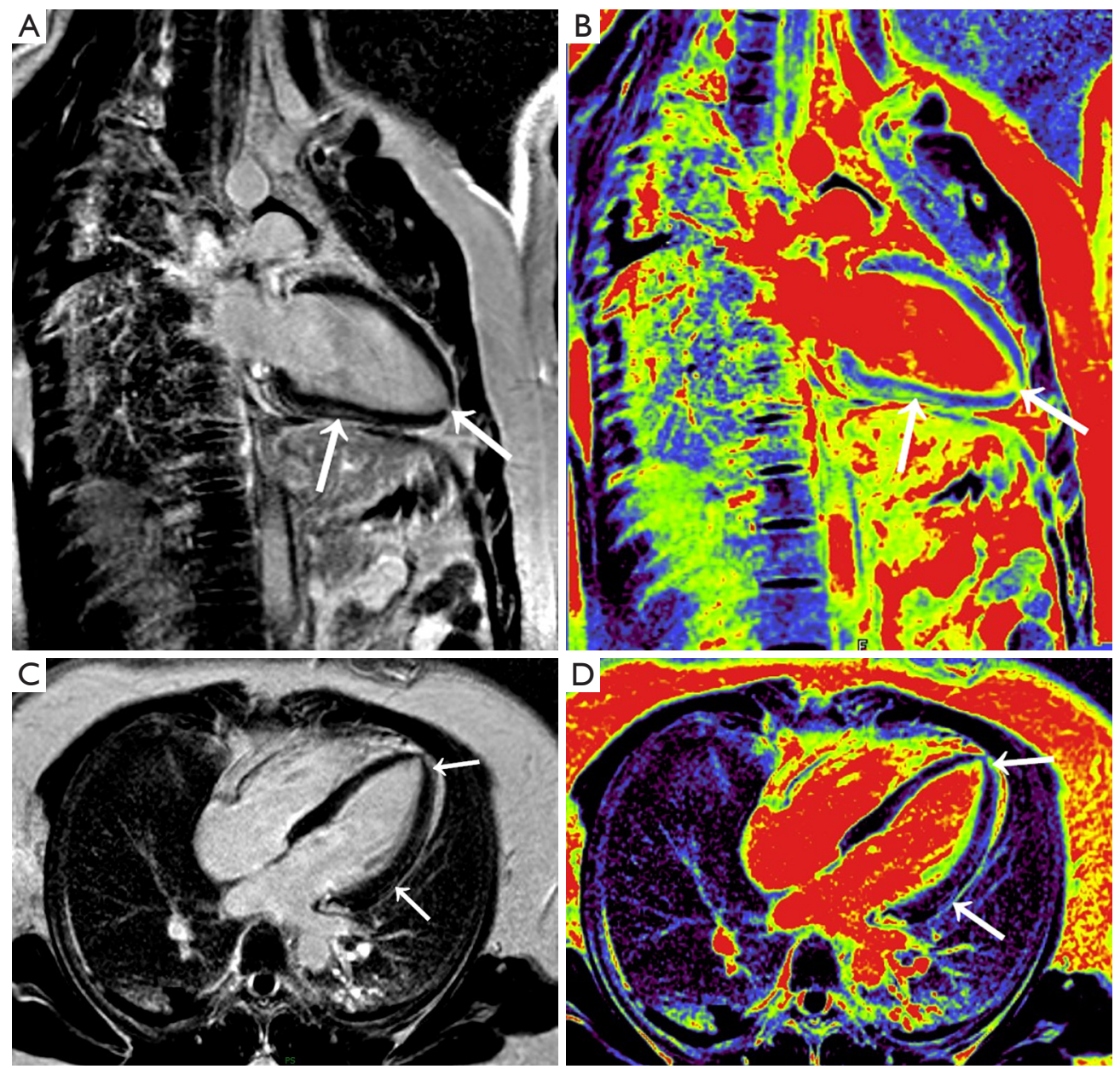

Figure 2 Original two-chamber (A), pseudo-color two-chamber (B), original four-chamber (C), and pseudo-color four-chamber (D) delayed-enhancement T1-weighted multishot gradient-echo IR magnetic resonance images of myocarditis in a 40-year-old woman. The band-like subepicardial LGE (arrows) of the left-ventricular lateral and inferior walls associated with nodular predominating in the apical segment can be seen. LGE, late gadolinium enhancement.

and LVESV are the first choice of parameters to reflect overall $\mathrm{LV}$ structure and function when the signal intensity of the 17th segment is increased on T2WI, EGE, or LGE images. Because the 4D method is based on the traditional 3D Simpson method, with the addition of short-axis cine images of the base, middle, and apex connected in series, it can be used to evaluate the systolic and diastolic function of the left ventricle more accurately and dynamically. We found that the independent parameter of LVEF based on the 4D method might have higher diagnostic efficiency than the independent parameter of LVESV and the combined parameter of LVEF-and-LVESV, so we recommend it as the first choice. If the Simpson method is used, the combined parameter of LVEF-and-LVESV may have higher diagnostic efficiency than the independent parameters of LVEF and LVESV, so it is recommended as the first choice. Taking the parameter of LVESV as an example, it was $64.24 \pm 18.48$ and $77.15 \pm 19.58 \mathrm{~mL}$ by the Simpson method and 4D method respectively, with large standard deviation, which may be related to the degree of myocardial fibrosis and the degree of LV decompensation. In addition, EKG in this study suggested that the incidence of atrial arrhythmia was higher than that of ventricular arrhythmia, which might indicate dysfunction of the left atrium (whether primary or secondary). Further study of left atrial function by CMRI is recommended for deep learning (18). 

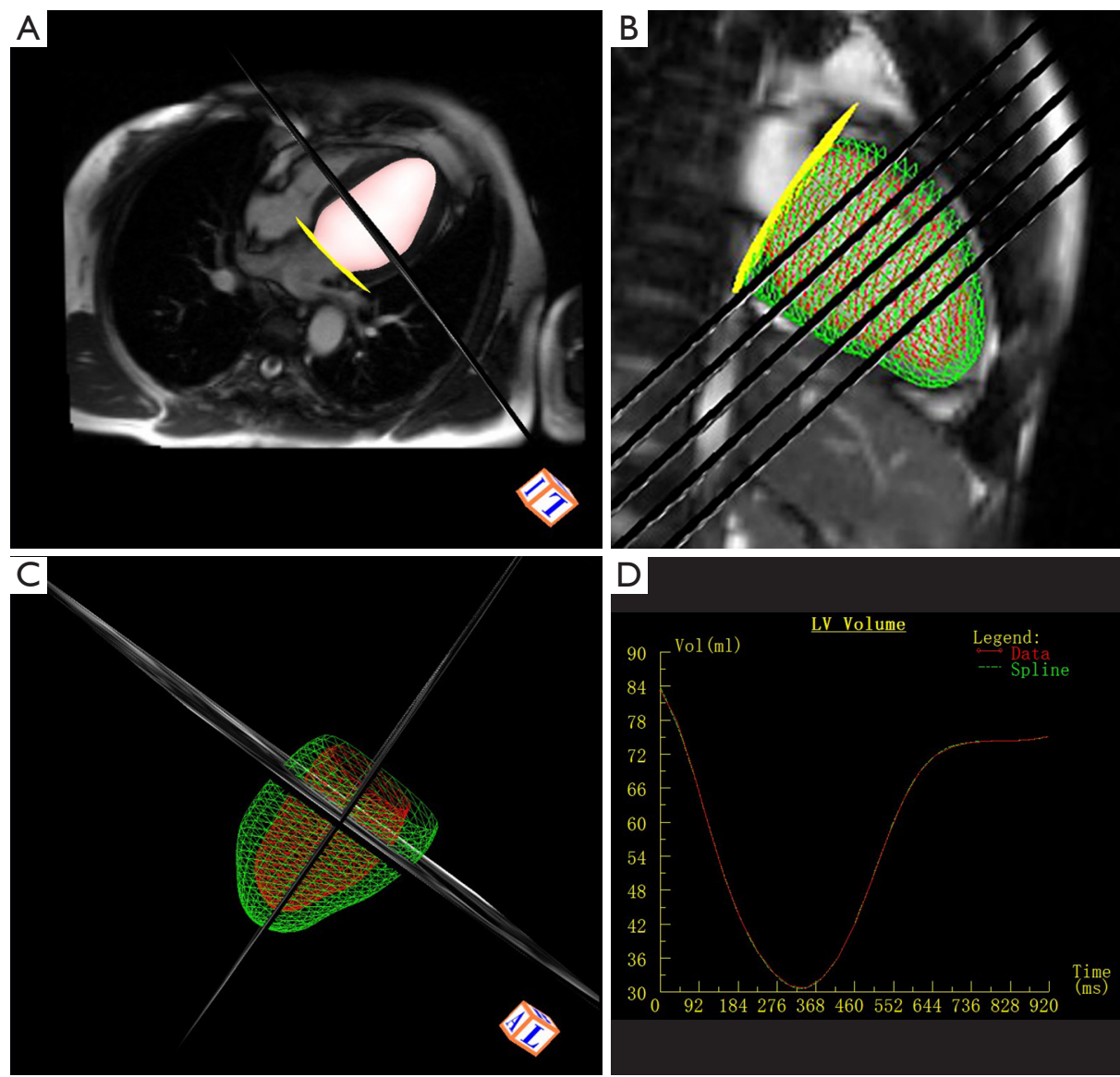

Figure 3 4D B-spline imaging and data reconstruction in a 28-year-old man with myocarditis. (A) Pink cone for blood pool measurement. (B) Series connection and recombination of short-axis with a simulation network of red endocardium and green epicardium. (C) Dynamic observation of left ventricular systole and diastole with $360^{\circ}$ rotation. (D) Left ventricular global volume-time curve.

Table 3 Comparison of left ventricular functional parameters between Simpson and 4D groups

\begin{tabular}{lcccc}
\hline & Simpson & 4D & F value & P value \\
\hline LVEF $(\%)$ & $41.76 \pm 11.16$ & $34.52 \pm 10.66$ & 4.841 & 0.033 \\
FS (\%) & $24.42 \pm 1.95$ & $24.26 \pm 2.51$ & 0.057 & 0.979 \\
LVEDD $(\mathrm{mm})$ & $45.74 \pm 4.85$ & $47.52 \pm 6.86$ & 3.149 & 0.328 \\
LVEDV $(\mathrm{mL})$ & $108.83 \pm 14.31$ & $116.50 \pm 14.34$ & 5.059 & 0.083 \\
LVESV $(\mathrm{mL})$ & $64.24 \pm 18.48$ & $77.15 \pm 19.58$ & 0.540 & 0.030 \\
LVMM $(\mathrm{g})$ & $58.07 \pm 5.92$ & $59.93 \pm 10.29$ & 3.021 & 0.466 \\
LVSV $(\mathrm{mL})$ & $44.59 \pm 10.01$ & $39.34 \pm 10.03$ & 0.012 & 0.090 \\
CO $(\mathrm{L} / \mathrm{min})$ & $3.09 \pm 0.64$ & $3.11 \pm 0.98$ & $2.64 \pm 0.38$ & 0.763 \\
CI (L/min/m2) & $2.75 \pm 0.41$ & & 0.387
\end{tabular}

LVEF, left ventricular ejection fraction; fraction shortening; LVEDD, left ventricular end-diastolic dimension; LVEDV, left ventricular end-diastolic volume; LVESV, left ventricular end-systolic volume; LVMM, left ventricular myocardial mass; LVSV, left ventricular stroke volume; $\mathrm{CO}$, cardiac output; $\mathrm{Cl}$, cardiac index. 

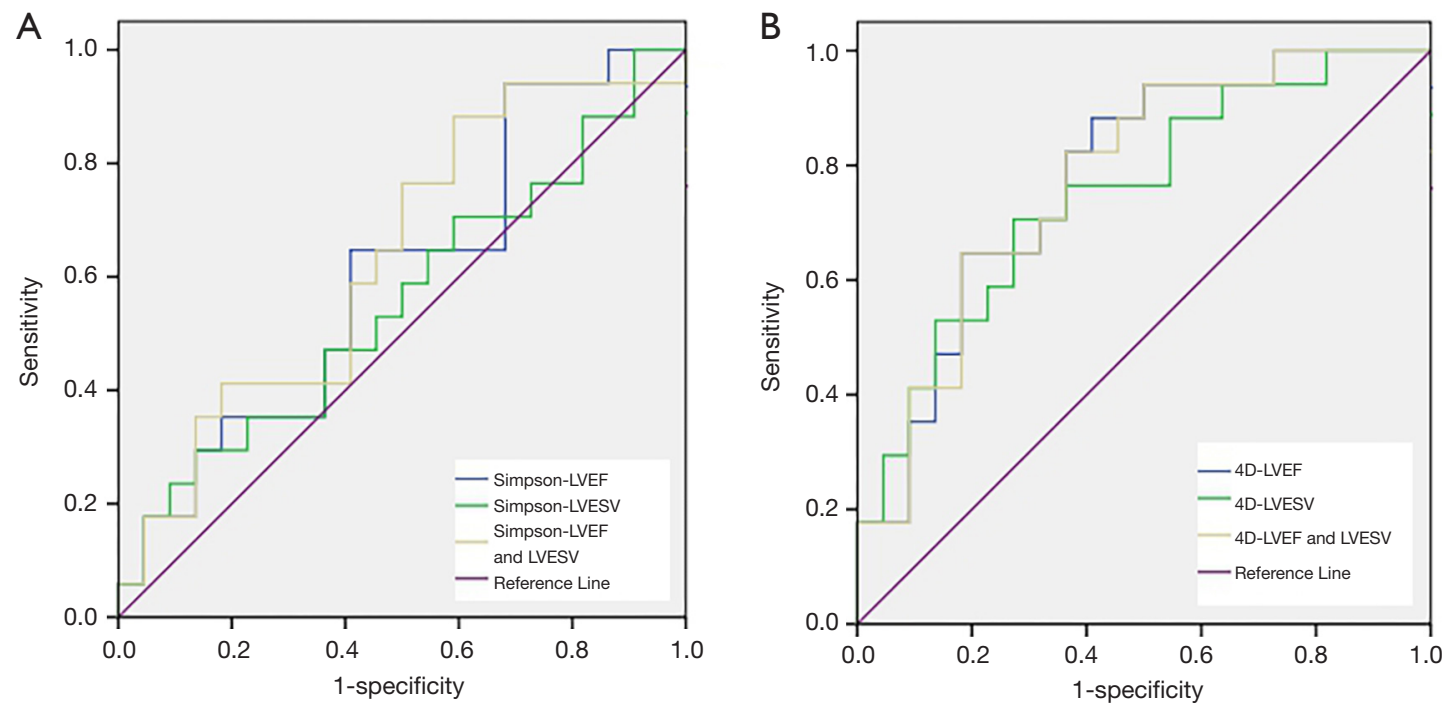

Figure 4 Receiver operating characteristics curve of independent and combined parameters with (A) the Simpson method and (B) the 4D B-spline method.

\section{Study limitations}

This was a small, observational study, which might have lacked the sufficient amount of data and thus urgently needs data from a large sample to support the conclusions. In early acute myocarditis, myocardial necrosis and interstitial fibrosis are not significant, so it is difficult to find abnormal delayed enhancement and significant changes in the LV function parameters. When drawing the endocardial and epicardial lines, the inner and outer rings might have been close to overlapping, which could lead to inaccurate recording of $L V$ function.

\section{Conclusions}

Compared with the Simpson method, the 4D method might be more effective in the diagnosis of apical-segmentinjured myocarditis. When the Simpson method is used, LVEF combined with LVESV might be the recommended parameter for comprehensive evaluation to improve diagnosis efficiency. When the 4D method is used, LVEF might be the preferred parameter for evaluation of $\mathrm{LV}$ function.

\section{Acknowledgments}

Funding: The study was supporting by the Scientific Research Project of Health Commission of Sichuan Province (no. 19PJ188) and the Special Research fund of the First Affiliated Hospital of Chengdu Medical College (no. CYFY2019YB03).

\section{Footnote}

Conflicts of Interest: Both authors have completed the ICMJE uniform disclosure form (available at http://dx.doi. org/10.21037/qims-20-287). The authors have no conflicts of interest to declare.

Ethical Statement: The study was approved by the Medical Ethics Committee of the First Affiliated Hospital of Chengdu Medical College (no. ChiCTR1800020447), and informed consent was taken from all the patients.

Open Access Statement: This is an Open Access article distributed in accordance with the Creative Commons Attribution-NonCommercial-NoDerivs 4.0 International License (CC BY-NC-ND 4.0), which permits the noncommercial replication and distribution of the article with the strict proviso that no changes or edits are made and the original work is properly cited (including links to both the formal publication through the relevant DOI and the license). See: https://creativecommons.org/licenses/by-nc-nd/4.0/.

\section{References}

1. Yajima T, Knowlton KU. Viral myocarditis: from the 
perspective of the virus. Circulation 2009;119:2615-24.

2. Adegbala O, Olagoke O, Akintoye E, Adejumo AC, Oluwole A, Jara C, Williams K, Briasoulis A, Afonso L. Predictors, Burden, and the Impact of Arrhythmia on Patients Admitted for Acute Myocarditis. Am J Cardiol 2019;123:139-44.

3. Mukai-Yatagai N, Haruki N, Kinugasa Y, Ohta Y, Ishibashi-Ueda H, Akasaka T, Kato M, Ogawa T, Yamamoto K. Assessment of myocardial fibrosis using T1mapping and extracellular volume measurement on cardiac magnetic resonance imaging for the diagnosis of radiationinduced cardiomyopathy. J Cardiol Cases 2018;18:132-5.

4. Friedrich MG, Marcotte F. Cardiac magnetic resonance assessment of myocarditis. Circ Cardiovasc Imaging 2013;6:833-9.

5. Friedrich MG, Sechtem U, Schulz-Menger J, Holmvang G, Alakija P, Cooper LT, White JA, Abdel-Aty H, Gutberlet M, Prasad S, Aletras A, Laissy JP, Paterson I, Filipchuk NG, Kumar A, Pauschinger M, Liu P. Cardiovascular magnetic resonance in myocarditis: A JACC White Paper. J Am Coll Cardiol 2009;53:1475-87.

6. Katritsis D, Hossein-Nia M, Anastasakis A, Poloniecki I, Holt DW, Camm AJ, Ward DE, Rowland E. Use of troponin-T concentration and kinase isoforms for quantitation of myocardial injury induced by radiofrequency catheter ablation. Eur Heart J 1997;18:1007-13.

7. Feintuch A, Zhu Y, Bishop J, Davidson L, Dazai J, Bruneau BG, Henkelman RM. 4D cardiac MRI in the mouse. NMR Biomed 2007;20:360-5.

8. Metaxas DN, Axel L, Qian Z, Huang X. A segmentation and tracking system for 4D cardiac tagged MR images. Conf Proc IEEE Eng Med Biol Soc 2006;2006:1541-4.

9. Segars WP, Lalush DS, Frey EC, Manocha D, King MA, Tsui BM. Improved Dynamic Cardiac Phantom Based on 4D NURBS and Tagged MRI. IEEE Trans Nucl Sci 2009;56:2728-38.

Cite this article as: Zhao XX, Yuan WF. The 4D B-spline method of calculating left ventricular functional parameters of cardiac MRI to evaluate myocardial injury of the apical segment in patients with myocarditis: a case-controlled observational study. Quant Imaging Med Surg 2020;10(11):2133-2143. doi: 10.21037/qims-20-287
10. Stalidis G, Maglaveras N, Efstratiadis SN, Dimitriadis AS, Pappas C. Model-based processing scheme for quantitative 4-D cardiac MRI analysis. IEEE Trans Inf Technol Biomed 2002;6:59-72.

11. Coatrieux JL, Hernández AI, Mabo P, Garreau M, Haigron P. Transvenous path finding in cardiac resynchronization therapy, 2005. International Workshop on Functional Imaging and Modeling of the Heart. 2005: 236-45.

12. Delhay B, Lotjonen J, Clarysse P, Katila T, Magnin IE. A dynamic 3-D cardiac surface model from MR images, 2005. Computers in Cardiology. 2005: 423-6.

13. Pattynama PM, Lamb HJ, van der Velde EA, van der Wall EE, de Roos A. Left ventricular measurements with cine and spin-echo MR imaging: a study of reproducibility with variance component analysis. Radiology 1993;187:261-8.

14. Moon JC, Lorenz CH, Francis JM, Smith GC, Pennell DJ. Breath-hold FLASH and FISP cardiovascular MR imaging: left ventricular volume differences and reproducibility. Radiology 2002;223:789-97.

15. Marghany M. Four-dimensional water detection in mars using spline algorithm. Int J Hydro 2018;2:607-11.

16. Chandrashekara R, Rueckert D, Mohiaddin R. Cardiac motion tracking in tagged MR images using a 4D B-spline motion model and nonrigid image registration. IEEE International Symposium on Biomedical Imaging: Nano to Macro. 2004;468-71.

17. Guo L, Guo H, Yuan Y, Zeng WY, Yuan SG, Zhou XW, Yang N, Ai LP. A Comparative Study of Left Ventricular Function Measured by 4D Cardiac Magnetic Resonance and 3D Cardiac Magnetic Resonance in Healthy Volunteers. Chinese Journal of Arteriosclerosis 2016;24:840-4.

18. Ghodrati V, Shao J, Bydder M, Zhou Z, Yin W, Nguyen $\mathrm{KL}$, Yang Y, Hu P. MR image reconstruction using deep learning: evaluation of network structure and loss functions. Quant Imaging Med Surg 2019;9:1516-27. 